\title{
Benchmarking and library quality maturity
}

\author{
Frankie Wilson \\ Brunel University, Uxbridge, UK, and \\ J. Stephen Town \\ Defence College of Management and Technology Library, Cranfield University, \\ Shrivenham, UK
}

\begin{abstract}
Purpose - It remains unresolved from the literature whether benchmarking is a useful and appropriate tool for the library and information services sector. The aim of this research was to gather evidence to establish whether benchmarking provides a real and lasting benefit to library and information services.

Design/methodology/approach - The study investigated the long term effects of a benchmarking exercise on the quality level of three UK academic libraries. However, an appropriate framework for assessing the quality level of libraries is not present in the literature, and it was therefore necessary for such a framework to be developed. This article describes and provides initial characterisation of the framework developed - the Quality Maturity Model (QMM).

Findings - The evidence from the investigation showed that the two libraries which were at stage one on the QMM before the benchmarking exercise remained there; and the library which scored at the penultimate level, level four, before benchmarking, was, four years afterwards, at level five. The tentative conclusion drawn were that benchmarking may only be appropriate for organisations with a existing high level of quality maturity. Much further work is proposed.

Originality/value - The research provides evidence which establishes whether benchmarking provides a real and lasting benefit to library and information services.
\end{abstract}

Keywords Benchmarking, Quality, Libraries, United Kingdom Paper type Research paper

\section{Introduction}

Benchmarking has been a widely used tool in business and industry for 25 years. It has been credited with enabling companies to survive changes that would otherwise have pushed them into crisis, by improving quality (Camp, 1989). It is usually linked to Total Quality Management (TQM) - an approach to quality that embraces the whole organisation and takes the customer as its starting point (Oakland, 1995).

The potential benefits of benchmarking for library and information services have been widely acknowledged. Library and information services have operated in an environment that demands a demonstration of quality since the 1990s. Although libraries have a very strong background of performance measurement, these 
measures do not address the needs of stakeholders for quality assessment. Numerous authors (e.g. Cheetham, 1993; Shaughnessy, 1993; Kinnel and Garrod, 1995; Town, 1995) advocate the application of benchmarking to libraries, joined by SCONUL in $\mathbf{2 0 0 0}$ with the publication of a benchmarking manual.

Benchmarking has been applied in academic libraries in the UK since 1995, with successful examples documented in the literature (Town, 2000). However, the adoption of benchmarking by libraries and information services has not been widespread. Kinnell and Garrod (1995) put the figure at 7 per cent of UK academic libraries.

Some authors have suggested that benchmarking is not appropriate for the library and information sector, proclaiming it "Just another management fad" (Brockman, 1992). Suggested barriers to the take up of benchmarking include the vocabulary of management techniques, the level of commitment required, the long-term nature of the process, and fears about professionalism (St. Clair, 1997).

Benchmarking involves a non-trivial investment in time and resources. An IBC study in 1992 (cited in Macdonald \& Tanner, 1988, p.19) surveyed 80 leading American organisations, and concluded that an average benchmarking study lasts for six months, occupies 25 per cent of team members' time, and costs $£ 45,000$.

Given this significant investment required to apply the technique, decision makers require unequivical evidence that establishes whether benchmarking provides a real and lasting benefit to library and information services.

\section{The investigation}

To try to determine whether benchmarking really does improve the quality of a library, or whether it can be considered just another passing fad, the effects of benchmarking exercises in three academic libraries was investigated. These libraries had undertaken a benchmarking exercise four years before this investigation. The aim was to determine whether the benchmarking exercise had provided real and lasting benefits to each of the participating libraries.

The research employed a qualitative case study approach, and the method used was a combination of documentary analysis and structured interviews. The documentary analysis was undertaken on both strategic and procedural documents, from the public domain, internal to the institutional, and internal to the library. Interview participants were a sample of staff from each service, reflecting all levels within the library and different periods of employment.

\section{A framework for measuring the quality process}

The key to the investigation was to judge whether the benchmarking exercise had improved the quality level of the three libraries. However, the quality 
literature does not give a framework for the measurement of the quality processes in an organisation. There is much in the literature (e.g. Hradesky, 1995; Oakland, 1993, 2003; Tenner and De Toro, 1992) about frameworks for measuring the quality of a product, process or service, but no framework for measuring the quality of the quality-process. The ISO9000 standard is similarly concerned with product quality, with the focus on the technical system of procedures and work instructions (Pike and Barnes, 1994) and not the achievement of a quality culture. Even a book entitled Achieve total quality (Hutchins, 1992) gives no indication of how to tell when an organisation has reached this goal!

The literature gives only one framework for measuring the quality culture of an organisation. The Software Engineering Institute developed the Capability Maturity Model (CMM) as a method of judging the quality of the software processes of an organisation (Paulk et al., 1993; Paulk, 1994). It is intended to be an evolutionary path to help organisations improve the quality maturity of their software processes from "ad hoc, chaotic processes to mature, disciplined software processes" (Paulk, 1994, p.3). The CMM has five levels with descriptive criteria for the attainment of each level. Organisations at level one are "low quality"; organisations at level five are "high quality".

This model is not as inappropriate for use in libraries as it might at first appear. During the print era, where libraries were "storehouses", quality could be assured by the rigorous application of standardised methods and work practices. However, with the move to the electronic era much library work is undertaken in the form of projects, so such methods are too inflexible to ensure high quality is maintained. The software industry is built around project work, and so methods that ensure high quality there are likely to transfer to the new library environment.

In order to assess the level of impact of benchmarking on the respective library services, a framework for measuring the general quality of an organisation had to be developed. The model created for the research was an adapted version of the CMM. This new model (retitled the Quality Maturity Model (QMM)) allows the quality maturity of a library service to be measured on a five-step scale. Each point on the scale has a general description of the level of quality in the organisation, with a list of specific attributes relevant to that level. The benefit of the model is that it translates qualitative descriptions into a quantitative score of progress towards the goal of a culture of continuous improvement.

The characterisations of the five levels of the QMM are a follows:

(1) Level 1 - initial.

The quality management process is ad hoc, and occasionally even chaotic. Few processes are defined, and success depends on individual effort and heroics:

- quality is achieved in an ad hoc way;

- customer satisfaction is reactive and unpredictable; 
- quality depends on the capabilities of individuals, and varies with their innate skills, knowledge and motivations; and

- training for quality is ad hoc and reactive to an ability to undertake a specific task adequately.

\section{(2) Level 2 - repeatable}

Basic quality management processes are established. The necessary management processes are in place to repeat earlier quality levels:

- quality policies, and procedures to implement these policies, are established;

- there are effective management processes to allow the organisation to repeat earlier success in customer satisfaction;

- such management processes are practised, documented, enforced, trained, measured, and able to improve; and

- training for quality is provided as a programme of training for specific work tasks, and / or is reactive to events.

\section{(3) Level 3 - defined}

The quality processes are documented and standardised. All work derives from the organisational strategy:

- there is a defined, documented organisational strategy, from which all work processes are derived;

- there is an organisation-wide understanding of the activities, roles, and responsibilities of each member of the organisation, and how they fit into the organisational strategy; and

- training for quality is a cycle of training needs assessment and programme provision.

\section{(4) Level 4 - managed}

Detailed measures of the quality process are collected. The quality process is quantitatively understood and controlled:

- quality measures are part of every documented work process;

- these measurements form the basis for evaluating products and processes;

- changes are implemented to improve the quality of services, products and processes;

- the organisation sets quantitative goals for quality and customer satisfaction; and

- training for quality is a cycle of training needs assessment, programme provision, and measurement of the effectiveness of the programme.

\section{Level 5 Optimising}

Continuous quality improvement is enabled by quantitative feedback and from piloting innovative ideas:

- the entire organisation is focussed on continuous improvement in every service, product and process; 
- all staff are encouraged to continuously improve themselves and their work;

- the organisation is able to identify weaknesses, and the means to strengthen the process, proactively with the goal of preventing problems.

- innovations that exploit the best practices are identified and transferred throughout the organisation; and

- training for quality is focussed on preparing staff for future organisational requirements.

\section{Applying the framework}

The evidence gathered from the interviews and documentary analysis was mapped against the model.

An indicative selection of the evidence indicating a level one library and a level five library is given below (direct quotes from interviews are shown in quotation marks; direct quotes from documentary analysis are shown in quotation marks and italics):

Level one

"Since the active and energetic chair of the [quality services group] left, the group has gone into abeyance." This indicates that quality is ad hoc, and dependent on the innate qualities of the staff.

"how far the QSG could go if a particular group was not sufficiently motivated to produce results" was not resolved for the group, and demonstrates the lack of managerial support for quality processes.

The library has a strategic plan, but documented work processes unrelated to it and do not include quality processes.

The library does not make changes to improve quality, but for PR: "[small changes will] improve the customer's perception of the service and ... demonstrate the library is responsive to feedback".

Staff are not encouraged to continuously improve, but asked: "can you think of any improvement which could realistically be considered?" and to:

"Separate out things which can and cannot be achieved".

Training is ad hoc and responsive: "There is much [training] available - if you want it you just need to ask for it." "there is support for staff to develop if they want to". "If you don't want to [improve] and want to stay at the same level, they are quite happy with that".

\section{Level five}

Continuing improvement bonuses are available for all staff, related to performance indicators against personal and team targets, which are linked to the strategic aims.

The library, and all its staff, continually strive to improve: "The library will seek to compare its performance systematically with that of other similar universities and emulate best practice in the sector" "there is lots of freedom to develop your own 
work area, as long as what you do is within the strategic aims of the library." "We can contribute ideas [for improvement] quite freely." "Though communication

within the service was commended ... we do still feel there is room for improvement".

Training is focused on preparing staff for future challenges: "The development of our staff is a major investment in the future success of the University" "They see [professional development] as a benefit to the whole service. The staff are developing themselves and then give a better service to the user."

\section{Results}

The results of the research showed that two of the three libraries were at level one on the QMM before the benchmarking study took place, and remained at that level after the study, four years later. Benchmarking has not had any impact on the long-term quality level of these libraries. This finding was supported by the evidence from interviewees at these libraries, who were explicitly asked: "What effects did the benchmarking study have on the library?" One interviewee at library 3 replied "none at all" and another interviewee at library 1 responded:

I do not think that there was anything radical that we did that came out of the whole thing.

Both of these libraries mentioned a "Hawthorne effect" (where an individual's behaviour alters because they know they are being studied) for the duration of the study, but not beyond.

However, the results also showed that one of the libraries scored at level four on the QMM before the benchmarking study took place, and scored at level five after the study, four years later. Benchmarking did have a real and long lasting beneficial effect on the quality level of this library. Again, this was supported by evidence from the interviewees:

We have that culture now of continuous improvement. Whether it was benchmarking specifically that set it off I don't know, or whether we always had it to some degree, but it acted as a catalyst $\ldots$ and it did make it much more systematic than it had been in the past.

The investigation suggests that benchmarking can have a beneficial long-term effect on library and information service quality. However, it also indicates that this effect may only occur in library and information services that already have a pre-existing quality approach (as shown by a high score on the QMM).

This finding is consistent with the literature: An Ernst \& Young survey (Ernst \& Young and the American Quality Foundation, 1993) reported that benchmarking was only of benefit to higher performing organisations, and suggested that lower performing organisations should concentrate on the basics. Kinnell and Garrod (1995) suggest that benchmarking can only be successfully implemented in libraries that have already begun to implement quality management practices. 
This finding is consistent with the QMM itself. Organisations at the lower levels of quality maturity do not have an organisational culture that enables them to make use of benchmarking. There must be a strong management commitment to all four elements of benchmarking (survey, comparison, understanding, implementation, e.g. Camp, 1989) for an exercise to be successful (St. Clair, 1993; Codling, 1998; Macdonald and Tanner, 1998). This will only be the case in organisations where the goal of achieving quality underpins all work policies i.e. organisations that score 4 or above on the QMM.

That a benchmarking exercise can result in an organisation moving from level 4 on the QMM to level 5 is consistent with the proposal that benchmarking leads to the creation of a learning organisation (McNair and Leibfried, 1992; Karlof and Ostblom, 1993; Zairi and Leonard, 1994). The essential difference between level 4 and level 5 is that at level 4, improvement in the quality of a process is the responsibility of the manager of that process. At level 5 , improvement in the quality of a process is the responsibility of every member of staff in the organisation. Such staff empowerment can only be achieved successfully in a learning organisation.

The finding that benchmarking is only successful in organisations with high quality maturity may also provide a possible indication of why benchmarking has been used with widespread success in industry, but has been felt by some to transfer unsuccessfully to library and information services. The competitive nature of industry means that a successful company could be expected to have implemented a succession of quality approaches, culminating in TQM, before conducting a benchmarking exercise i.e. industry started at the bottom and worked upwards in stages until reaching a high QMM level before applying benchmarking, and so benefited from it. Library and information services have do not have the same history of a quality approach, and so were not at the required level of maturity when applying benchmarking - they jumped straight into using a tool appropriate for high quality maturity organisations when they just weren't ready. No wonder it failed.

\section{Further work}

The research described in this paper was a small exploratory study into the unknown. The research investigated three academic libraries and used a new reference model as a framework for measurement. From the results of these investigations a number of wide-ranging conclusions have been postulated. Although these conclusions do appear to be consistent with the known literature, they can be viewed as nothing more than very tentative until much further research has been undertaken.

The proposed QMM must be tested and further characterised. Research is needed to investigate whether the model is able to uniquely and consistently determine the quality level of library and information services at all five levels. 
Research is also needed to describe the properties of organisations at all five levels, and so enable fuller characterisation of the levels. This might in turn lead to an instrument to permit libraries to diagnose their own individual levels of maturity, and also to recommending specific quality management techniques (in addition to benchmarking) which are appropriate to a particular level of quality maturity.

This research investigated libraries that were at level 1 or level 4 prior to undertaking a benchmarking exercise. The conclusions propose that benchmarking will only result in real and lasting benefits for libraries at level 4 on the QMM before the exercise. In order for the model, and so the conclusions, to be valid, this must be the point of demarcation between the two outcomes. Further research is needed to determine the effects of a benchmarking study on libraries that scored 2, 3 and 5 on the QMM prior to their benchmarking exercise.

The initial QMM score for the three libraries in this research was obtained retrospectively, i.e. some time after the benchmarking exercise had taken place. For the QMM to be a useful predictor of whether the investment in a benchmarking exercise would be justified in term of improvements, a priori testing of the quality maturity level of a library before a benchmarking exercise is necessary. Research into whether the QMM is predictive is likely to be beneficial to the sector.

\section{Conclusion}

There is still much research to be done, but a tentative conclusion can be drawn that benchmarking should be viewed as a tool for organisations at a high level of quality maturity. When used appropriately it can have real and long-lasting benefits, particularly in driving a culture of continuous improvement. When used inappropriately it is a waste of time and effort. We should remember not to run before we can walk.

\section{References}

Brockman, J.R. (1992), "Just another management fad? The implications of TQM for library and information services". ASLIB Proceedings, Vol. 44 Nos. 7/8, pp. 283-288.

Camp, R.C. (1989), Benchmarking: the search for industry best practices that lead to superior performance. American Society for Quality Control Press, Milwaukee, III.

Cheetham, D.L. (1993), "The potential of benchmarking for higher education libraries. Paper given at the Training Together 3 meeting held at Manchester Metropolitan University, 22 April 1993". COPOL Newsletter, Vol. 62, pp.67-73.

Codling, s. (1998), Benchmarking. Gower, Aldershot. 
Ernst \& Young and the American Quality Foundation. (1993), Best practices report: An analysis of management practices that impact performance. Ernst and Young, New York, NY.

Hradesky, J. (1995), Total Quality Management Handbook. McGraw-Hill, New York, NY.

Hutchins, D. (1992), Achieve total quality. Director books, Hemel Hempsted.

Karloff, B. and Ostblom, S. (1993), Benchmarking: a signpost to excellence in quality and productivity. Wiley, Chichester.

Kinnell, M. and Garrod, P. (1995), "Benchmarking and its relevance to the library and information sector: interim findings of 'best practice benchmarking in the library and information sector', a British Library Research and Development project". Proceedings of the Northumbria international conference on performance measurement in libraries and information services. Information North, Newcastle-upon-Tyne pp.159-171.

Macdonald, J. and Tanner, S. (1998), Understanding benchmarking in a week. Rev ed., Hodder \& Stoughton, London.

McNair, C.J. and Leibfried, K.H.J. (1992), Benchmarking: a tool for continuous improvement. Wiley, New York, NY.

Oakland, J.S. (1993), Total quality management: the route to improving performance. $2^{\text {nd }}$ ed., Butterworth-Heinemann, Oxford.

Oakland, J.S. (1995), Total quality management: text with cases. ButterworthHeinemann, Oxford.

Oakland, J.S. (2003), Total quality management: text with cases. $3^{\text {rd }}$ ed., Butterworth-Heinemann, Oxford.

Paulk, M.C. (1994), A comparison of ISO 9001 and the Capability Maturity Model for software. CMU/SEI-94-TR-12. Software Engineering Institute, Pittsburgh, PA.

Paulk, M.C., Weber, C.V., Garcia, S.M., Chrissis, M.B. and Bush, M. (1993), Key practices of the Capability Maturity Model, version 1.1. CMU/SEI-93-TR-025. Software Engineering Institute, Pittsburgh, PA.

Pike, J. and Barnes, R. (1994), TQM in action: practical approach to continuous performance improvement. Chapman \& Hall, London. 
Shaughnessy, T.W. (1993), "Benchmarking, total quality management and libraries". Library administration and management, Vol. 7 No. 1, pp.7-12.

St. Clair. G. (1993), The future challenge: management and measurement. Special libraries, Vol. 84 No. 2, pp.151-154.

St. Clair, G. (1997), Total quality management in services. Bowker Saur, London.

Tenner, A.R., and De Torro, I.J. (1992), Total quality management: three steps to continuous improvement. Addison-Wesley, Wokingham.

Town, J.S. (1995), "Benchmarking and performance measurement". Proceedings of the Northumbria international conference on performance measurement in libraries and information services. Information North, Newcastle-upon-Tyne, pp.83-88.

Town, J.S. (2000), "Benchmarking: strife, theft or communion?" Proceedings of the Northumbria international conference on performance measurement in libraries and information services. Information North, Newcastle-upon-Tyne, pp.81-88.

Zairi, M. and Leonard, P. (1994), Practical benchmarking: the complete guide. London: Chapman \& Hall. 